\title{
Mathematikforschung im Audio-Podcast Modellansatz
}

\author{
Sebastian Ritterbusch und Gudrun Thäter
}

Seit Oktober 2013 reden wir mit Schülern, Studierenden und Kollegen über ihre Arbeit: darüber, woran sie gerade „tüfteln“. Dabei beschränkt sich der Kollegenkreis nicht nur auf Forscherinnen und Forscher am Karlsruher Institut für Technologie (KIT). Oft ergibt sich die Gelegenheit zum Gespräch am Rande von Tagungen oder mit Gästen, die für einen Vortrag oder einen Forschungsaufenthalt an die KIT-Fakultät für Mathematik nach Karlsruhe gekommen sind. Oder Mathematikerinnen und Mathematiker aus Industrieprojekten finden sich bereit, ihre Zeit für ein Gespräch zur Verfügung zu stellen. Manche Gäste werden eigens nach Karlsruhe eingeladen, oder einer von uns macht sich auf den Weg zu ihnen. Die Gespräche (im Schnitt drei pro Monat) werden aufgezeichnet und regelmäßig als Episoden im Audio-Podcast „Modellansatz“ (http://modellansatz.de) veröffentlicht.

Podcasts sind vergleichbar mit Radiosendungen, indem sie Menschen zum Zuhören einladen. Allerdings sind sie frei von einigen „Fesseln“ des Radioformats: Erstens gibt es für Podcast-Episoden in der Regel keine vordefinierte Länge und zweitens werden sie so aufbereitet, dass man sie mit einem Programm, meist einer App, abonnie- ren und dann abspielen kann, wenn man gerade Zeit dafür hat. Das kann man mit einem mp3-Spieler oder dem Handy mobil tun oder mit dem aufgeklappten Laptop oder Tablet an einem festen Ort. So versüßen diese Gesprächsepisoden für viele den Weg zur Arbeit oder in die Schule. Die Hörer erfahren beispielsweise, wie Mathematik in der Abschnittskontrolle auf Autobahnen oder in der Migräne-Forschung zum Einsatz kommt, oder welche Themen im ersten Semester „Höhere Mathematik“ in den Ingenieurstudiengängen des KIT behandelt werden und wie man sich am besten auf die Klausur vorbereitet.

Die Gespräche gehen in die Tiefe und man erfährt nicht nur viel über die Wissenschaft, sondern auch wie die Forscherinnen und Forscher überhaupt auf neue Ideen kommen und die Welt um sich herum sehen - und das aus erster Hand. Oft sprechen die Gesprächspartner auch darüber, warum sie diesen Beruf gewählt haben, wieso sie ihre Arbeit so faszinierend finden und wie sich ihr Bild von Mathematik und ihrem Fachgebiet darin entwickelt hat. Damit wird stückweise ein Mosaikbild geschaffen, das einerseits den Studiengang Mathematik und andererseits das Berufsbild Mathematikerin bzw. Mathematiker leben-

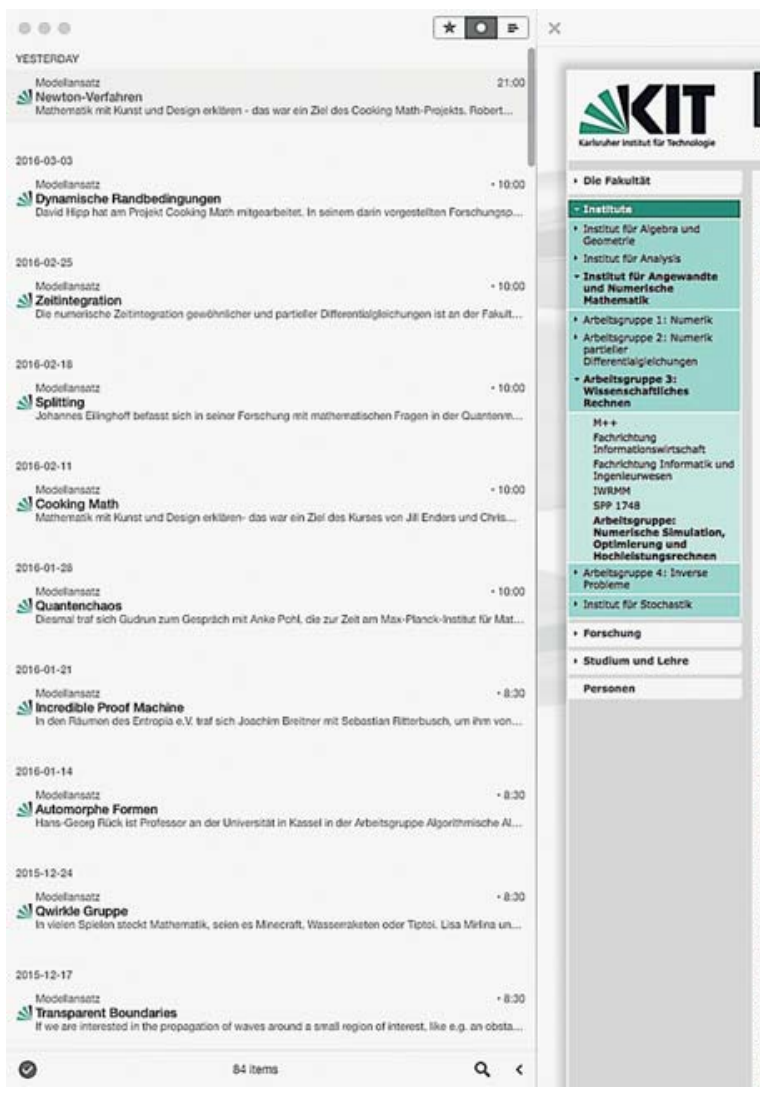

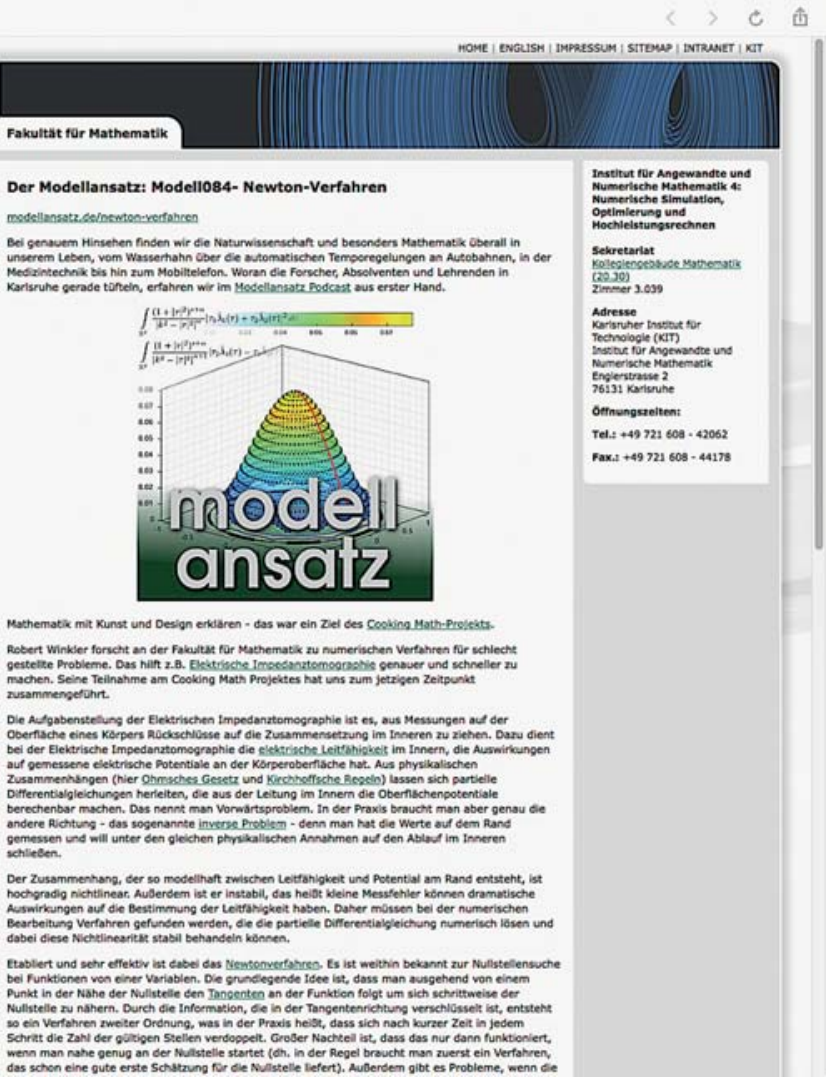




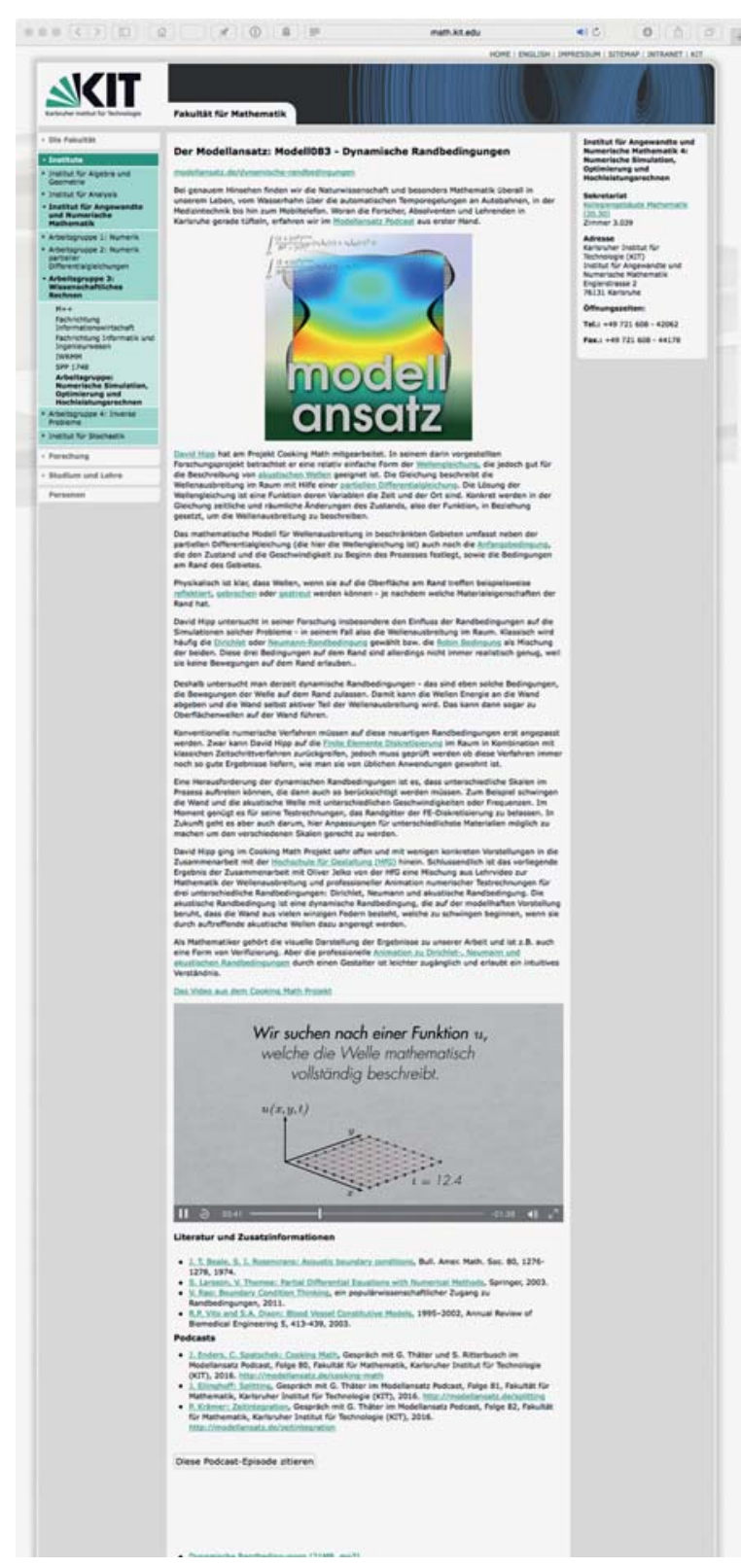

Zusammenfassung und weiterführende Informationen zum Podcast 83: „Dynamische Randbedingungen“

dig und anschaulich werden lässt. Nebenbei zeigt sich, wie viele Frauen in diesem Berufsfeld sehr erfolgreich und zufrieden arbeiten, denn fast die Hälfte der Episoden behandelt Projekte von Frauen. Die Gespräche dauern so lange, bis das Thema hinreichend erkundet ist. Das können 20 Minuten oder auch drei Stunden sein.

Für jede Episode wird überdies eine Webseite gestaltet, auf der neben dem Audioformat auch noch zusammenfassende Informationen zum Inhalt des Gesprächs zu finden sind sowie Links zu den zugehörigen mathematischen Veröffentlichungen, Erklärungen von Fachbegriffen und Methoden und weiterführende Literatur. Hier finden sich auch weitere Abbildungen, Videos und Simulationen. Damit haben alle Personen, deren Interesse an dem The- ma durch den Podcast geweckt wurde, die Möglichkeit, sich weiter zu informieren und relevante Quellen aus erster Hand zu studieren. Der Kontakt für direkte Nachfragen ist über E-mail, Twitter @modellansatz und Facebook sehr niedrigschwellig und einfach. Der Podcast ist über diese Kanäle auch sehr gut mit anderen Podcasts vernetzt, die über Wissenschaft berichten.

Die Nachfrage nach dem Podcast Modellansatz und dem Thema Mathematik ist sehr groß: Im Juni 2015 wurde die Marke von 100000 Downloads überschritten, Anfang August waren es dann schon 120000 . Wären die Folgen dreiminütige Youtube Videos, entspräche dies mehr als 2 Millionen Klicks.

Da die Abschlussarbeiten nicht ,im Schrank verschwinden“, werden Absolventinnen und Absolventen auf Bondingmessen auf den Podcast angesprochen und bei $A b$ schlussvorträgen zu Master- und Bachelorarbeiten in Mathematik mit Industriepartnern kommt am Schluss häufig die Frage: „Und wann können wir eine Podcastfolge dazu erwarten?"

Noch eindrücklicher ist vielleicht, dass sich der Podcast wie eine klassische Veröffentlichung verhält, und somit andere Forscher von neuen Ansätzen und Ideen erfahren. Ein Beispiel hierfür ist die Podcastfolge 43 zu Fußgängersimulationsmodellen. Menschenströme mit geeigneten Modellen zu simulieren, ist für die Planung großer Veranstaltungen Pflicht, damit Katastrophen wie bei der Love-Parade 2010 verhindert werden. Henrieke Benner hat das in Zusammenarbeit mit der Karlsruher PTVgroup in ihrer Bachelorarbeit erledigt und dafür sogar den RiMEA-Preis bekommen. Sie sprach mit uns über dieses spannende Thema und Forscher an der TU München wurden durch den Podcast auf neue Ansätze für eine Simulation zur Evakuierung der U-Bahn gebracht.

Dr. Sebastian Ritterbusch und PD Dr. Gudrun Thäter Karlsruhe Institute of Technology (KIT), Institut für Angewandte und Numerische Mathematik, Englerstraße 2, 76I3I Karlsruhe sebastian.ritterbusch@partner.kit.edu, gudrun.thaeter@kit.edu

Sebastian Ritterbusch, Jahrgang 1975. 19952002 Studium der Technomathematik an der TU Karlsruhe zum Diplom und Applied Mathematics zum M.Sc. an der UMass Amherst, USA, 2003-09 wissenschaftlicher Mitarbeiter TU Karlsruhe mit Promotion 2009, 2009-14 Leitung des Visualisierungslabs am EMCL des Karlsruher Institut für Technologie (KIT) und seit 2014 in Unternehmensberatung und Forschungskooperationen, sowie als Dozent an der Dualen Hochschule Baden-Württemberg (DHBW) tätig.

Gudrun Thäter, Jahrgang 1964. 1982-87 Studium der Mathematik an der TU Dresden, |987-9| Entwicklungsingenieurin Mikroelektronik, wissenschaftliche Tätigkeiten 1992-97 Uni Paderborn (dort Promotion 1996), 19972000 Uni Bonn, 2000-04 Uni Hannover (dort Habilitation 2004), 2004-08 TU Dortmund, 2008/09 Uni Heidelberg und seit 2009 Akademische Oberrätin am KIT.
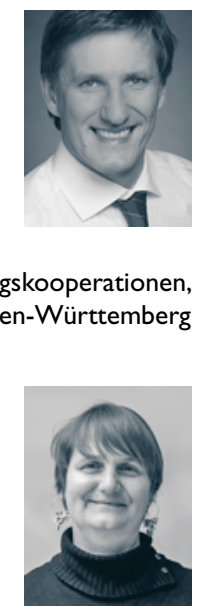